\title{
How to create public health Facebook posts based on Motivational Interviewing approach?
}

Pócs D, Department of Behavioral Science, Faculty of Medicine, University of Szeged.

\section{Objectives}

This research aimed at identifying which types of social media content could achieve higher engagement rate and encourage smokers' motivational language. Intervention contents were classified according to motivational interviewing (MI) strategies.

\section{Methods}

We categorized the included 701 Facebook posts ( $N=701)$ into five

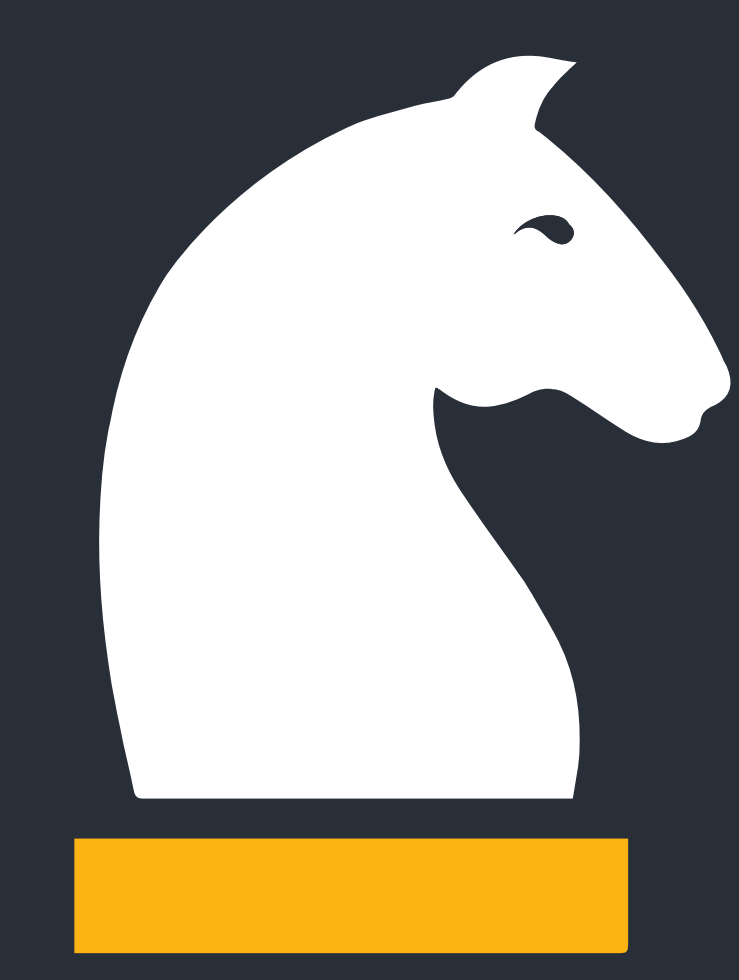
different groups according to specific MI strategies. Entertaining and informative strategies were in the control group. Facebook users' interactions were the primary outcomes (engagement rate, negative feedback, and fan-total reach ratio). The comments reflecting smokers' motivational language were the secondary outcomes (change talk and sustain talk).

\section{Results}

MI strategies achieved significantly higher engagement rate, higher fan-total reach ratio, and evoked more change talk. "Elaborating change talk" strategies elicited considerably more change talk. "Affirming change talk" strategies obtained higher fantotal reach ratio and generated significantly more change talk. "Relational MI" strategies achieved significantly higher engagement rate.

\section{Conclusions}

This study offers an important insight into the Facebook post creating for public health professionals who design Facebook-based interventions.

Strategies for Creating Facebook Posts that Can Increase the Engagement Rate:

- "Motivational Interviewing" strategies

- "Building Partnership" strategies

- "Expressing Empathy" strategies

Strategies for Creating Facebook Posts that Can Reach More Fans:

- "Motivational Interviewing" strategies

- "Affirming Change Talk" strategies

- "Reflecting Change Talk" strategies

Strategies for Creating Facebook Posts that Can Support Smoking Cessation:

- "Motivational Interviewing" strategies

- "Elaborating Change Talk" strategies

- "Affirming Change Talk" strategies 